\title{
AMALGAMATED PRODUCTS OF SEMIGROUPS: THE EMBEDDING PROBLEM
}

\author{
BY \\ GERARD LALLEMENT
}

\begin{abstract}
A necessary and sufficient condition for a semigroup amalgam to be embeddable is given. It is in the form of a countable set of equational implications with existential quantifiers. Furthermore it is shown that no finite set of equational implications can serve as a necessary and sufficient condition. Howie's sufficient condition (see [5]) is derived as a consequence of our main theorem.
\end{abstract}

1. Introduction. Let $\left\{S_{i} ; i \in I\right\}$ be a family of semigroups, and suppose that for each $i \in I$ there exists a homomorphism $h_{i}: U \rightarrow S_{i}$ from a semigroup $U$ into $S_{i}$. Consider the set $\Sigma=\left\{(x, i): i \in I, x \in S_{i}\right\}$, sum of the sets $S_{i}$, and construct the semigroup $S$ presented by

$$
\begin{aligned}
S=\left\langle\Sigma ;(x, i)\left(x^{\prime}, i\right)=\right. & \left(x x^{\prime}, i\right) \text { for every } i \in I, x \text { and } x^{\prime} \in S_{i}, \\
& \left.\left(h_{i}(u), i\right)=\left(h_{j}(u), j\right) \text { for every } i, j \in I, u \in U\right\rangle .
\end{aligned}
$$

Then $S$ is called the product of the family $S_{i}$ amalgamated by $U$ and is denoted by $\Pi_{i \in I}^{*} U S_{i}$. If $\varphi$ denotes the canonical homomorphism from the free semigroup on the alphabet $\Sigma$ onto $S$, then $\varphi_{i}$ defined by $\varphi_{i}(x)=\varphi((x, i))$ is a homomorphism from $S_{i}$ into $S$, and $h: U \rightarrow S$ defined by $h(u)=\varphi\left[\left(h_{i}(u), i\right)\right]$ is independent of $i$. Indeed, $\left\{S ; \varphi_{i}: S_{i} \rightarrow S\right\}$ is an initial object in the category whose objects are $\left\{T ; f_{i}: S_{i} \rightarrow T\right\}$ with $f_{i} \circ h_{i}$ independent of $i$.

Herein, we consider a family $\left\{S_{i} ; i \in I\right\}$ of semigroups and mappings $h_{i}$ : $U \rightarrow S_{i}$ that are injective homomorphisms for all $i \in I$. Under these conditions the system $\left[S_{i} ; U ; h_{i}\right]_{i \in I}$ is called a semigroup amalgam $[2, \mathrm{p} .138]$, and the set $G=\bigcup_{i \in I} \bar{S}_{i}$ where $\bar{S}_{i}=\left[S_{i} \backslash h_{i}(U)\right] \cup U$, with a partial binary operation defined in such a way that the natural mappings $\bar{S}_{i} \rightarrow S_{i}$ are isomorphisms, is called the groupoid of the amalgam $\left[S_{i} ; U ; h_{i}\right]_{i \in I}$. If there exist a semigroup $T$ and a mapping $\theta: G \rightarrow T$ such that $\theta$, when restricted to each $\bar{S}_{i}$, is an injective homomorphism, then the semigroup amalgam is said to be embeddable. The link between embeddable amalgams and amalgamated products is expressed in the next theorem:

Presented to the Society, January 16, 1974; received by the editors February 5, 1974. AMS (MOS) subject classifications (1970). Primary $20 \mathrm{M05}$.

Key words and phrases. Semigroup amalgam, amalgamated products of semigroups, embedding of amalgams, free products of semigroups. 
THEOREM 1.1 [2, THEOREM 9.31]. The following conditions are equivalent:

(i) The semigroup amalgam $\left[S_{i} ; U ; h_{i}\right]_{i \in I}$ is embeddable.

(ii) The canonical mapping of the partial groupoid $G$ of $\left[S_{i} ; U ; h_{i}\right]_{i \in I}$ into the product $\Pi_{i \in I}^{*} S_{i}$ is an embedding.

(iii) The homomorphisms $\varphi_{i}: S_{i} \rightarrow \Pi_{i \in I U}^{*} S_{i}$ have the properties

(a) $\varphi_{i}$ is an injection for every $i \in I$;

(b) $\varphi_{i}\left(S_{i}\right) \cap \varphi_{j}\left(S_{j}\right)=h(U)$ for $i, j \in I, i \neq j$, where $h=\varphi_{i} \circ h_{i}$.

So far, only sufficient conditions for a semigroup amalgam to be embeddable have been obtained. They are as follows:

Theorem 1.2 (Howie [5, Theorem 3.3]). Let $\left[S_{i} ; U ; h_{i}\right]_{i \in I}$ be a semigroup amalgam. If, for every $i \in I, h_{i}(U)$ is almost unitary (see [2, p. 144]) in $S_{i}$, then the amalgam is embeddable.

Theorem 1.3 (Bourbaki [1, Proposition 5, §7, p. I. 81]). Assume that, for every $i \in I, S_{i}$ is a monoid with identity $e_{i}$, that $U$ is a monoid and that $h_{i}$ : $U \rightarrow S_{i}$ are monoid homomorphisms (i.e. $h_{i}(e)=e_{i}$ for every $i \in I$ ). Assume further that for every $i \in I$ there exists a subset $P_{i}$ of $S_{i}$ containing $e_{i}$ such that the mapping $(u, p) \rightarrow h_{i}(u) p$ from $U \times P_{i}$ into $S_{i}$ is a bijection. Then every $x \in$ $\Pi_{i \in I}^{*} S_{i}$ can be written uniquely as $x=h(u) \Pi_{\alpha=1}^{n} \varphi_{i_{\alpha}}\left(p_{\alpha}\right)$ with $i_{\alpha} \neq i_{\alpha+1} \quad(1 \leqslant$ $\alpha<n), p_{\alpha} \in S_{i_{\alpha}}$ and $p_{\alpha} \neq e_{i_{\alpha}}(1 \leqslant \alpha \leqslant n)$.

In this theorem, the hypothesis that $(u, p) \rightarrow h_{i}(u) p$ is a bijection yields that $h_{i}$ is an injection. From the conclusion, it is easy to deduce that (iii) of Theorem 1.1 is satisfied, showing that the monoid amalgam $\left[S_{i} ; U ; \varphi_{i}\right]$ is embeddable.

The case of $h_{i}(U)$ being an ideal of $S_{i}$ is covered by the following

Theorem 1.4 (GRIllet-Petrich [3], LjaPin [6]). Let $\left[S_{i} ; U ; h_{i}\right]_{i \in I}$ be a semigroup amalgam. Suppose $h_{i}(U)$ is an ideal of $S_{i}$ for every $i \in I$. Then the amalgam is embeddable if and only if for every $s_{i} \in S_{i}, s_{j} \in S_{j}, u, u^{\prime}, u^{\prime \prime} \in U$, $s_{i} h_{i}(u)=h_{i}\left(u^{\prime}\right)$ and $h_{j}(u) s_{j}=h_{j}\left(u^{\prime \prime}\right)$ imply $h_{j}^{-1}\left(h_{j}\left(u^{\prime}\right) s_{j}\right)=h_{i}^{-1}\left(s_{i} h_{i}\left(u^{\prime \prime}\right)\right)$.

Note that, when expressed as a condition on the groupoid $G$ of the amalgam $\left[S_{i} ; U ; h_{i}\right]_{i \in I}$, the condition of Theorem 1.4 is simply $\left(s_{i} u\right) s_{j}=s_{i}\left(u s_{j}\right)$ for every $s_{i} \in \bar{S}_{i}, s_{j} \in \bar{S}_{j}, u \in U$.

Finally we mention an important result concerning the amalgamation of inverse semigroups.

TheOREM 1.5 (HALl [4]). A semigroup amalgam $\left[S_{i} ; U ; h_{i}\right]_{i \in I}$ with $S_{i}$ and $U$ inverse semigroups is embeddable in an inverse semigroup.

The main result of this paper (Theorem 3.3) consists of a necessary and 
sufficient condition for a semigroup amalgam $\left[S_{i} ; U, h_{i}\right]$ to be embeddable. The condition is that any system of equations of a certain nature (balanced system) over the semigroups $S_{i}(i \in I)$ implies another equation, or other equations (two at most) linked to the initial system. Briefly, our condition is in the form of a countable set of equational implications with existential quantifiers and with variables taken from card $I$ distinct sets. It is shown further (Theorem 4.4) that no finite set of equational implications can serve as a necessary and sufficient condition that a semigroup amalgam be embeddable. The techniques to prove our results are quite comparable to those used by Malcev ([7], [8]) to find necessary and sufficient conditions that a semigroup be embeddable in a group.

Theorem 1.2 and Theorem 1.4 above are derived as corollaries of Theorem 3.3, but to derive Proposition 2.4 (which generalizes Theorem 1.3) or Theorem 1.5 would require a deeper analysis of the structure of balanced systems. This indicates that, at least in its present form, the condition of Theorem 3.3 is not necessarily the shortest way of proving the embeddability of semigroup amalgams in particular situations.

2. Some notation and particular cases of embedding. When considering semigroup amalgams $\left[S_{i} ; U ; h_{i}\right]_{i \in I}$, we shall systematically assume that $S_{i} \cap S_{j}=\varnothing$ for $i, j \in I, i \neq j$. As a consequence, elements of the sum $\Sigma$ of the sets $S_{i}(i \in I)$ will be denoted $s_{i}$ or $s_{i}^{(k)}$, the superscripts being used for the purpose of distinguishing several elements in the same $S_{i}$. Similarly we shall write $U_{i}$ for $h_{i}(U)$, and elements of $U_{i}$ are denoted $u_{i}$ or $u_{i}^{(k)}$. Utilizing this notation we have $\Sigma=$ $\left\{s_{i}: s_{i} \in S_{i}, i \in I\right\}$, and denoting by $*$ the multiplication in the free semigroup over $\Sigma$, the presentation of $\Pi_{i \in I}^{*} S_{i}$ is written

$$
\left\langle\Sigma ; s_{i}^{(1)} * s_{i}^{(2)}=s_{i}^{(1)} s_{i}^{(2)}\left(i \in I, s_{i}^{(1)}, s_{i}^{(2)} \in S_{i}\right), u_{i}=u_{j}(i, j \in I)\right\rangle .
$$

The semigroup $\Pi_{i \in I}^{*} S_{i}$ presented by

$$
\prod_{i \in I}^{*} s_{i}=\left\langle\Sigma ; s_{i}^{(1)} * s_{i}^{(2)}=s_{i}^{(1)} s_{i}^{(2)} \text { for every } i \in I, s_{i}^{(1)}, s_{i}^{(2)} \in S_{i}\right\rangle
$$

is called the free product of the family $\left\{S_{i} ; i \in I\right\}$. An element $x \in \Pi_{i \in I}^{*} S_{i}$ can be written uniquely as $s_{i_{1}} * s_{i_{2}} * \cdots * s_{i_{m}}$ with $i_{k} \neq i_{k+1}$, for all $k, 1 \leqslant k \leqslant$ $m-1$, and the elements $s_{i_{k}}(1 \leqslant k \leqslant m)$ are called the syllables of $x$. Furthermore,

$$
\begin{aligned}
\left(s_{i_{1}} * \cdots * s_{i_{m}}^{(1)}\right) *\left(s_{j_{1}}^{(2)} * \cdots * s_{j_{n}}\right) & \\
& =s_{i_{1}} * \cdots * s_{i_{m}}^{(1)} * s_{j_{1}}^{(2)} * \cdots * s_{j_{n}} \quad \text { if } i_{m} \neq j_{1}, \\
& =s_{i_{1}} * \cdots * s_{i_{m-1}} *\left(s_{i_{m}}^{(1)} s_{j_{1}}^{(2)}\right) * s_{j_{2}} * \cdots * s_{j_{n}} \quad \text { if } i_{m}=j_{1} .
\end{aligned}
$$


Clearly $\Pi_{i \in I}^{*} S_{i}=\Pi_{i \in I}^{*} S_{i} / \rho$ where $\rho$ is the congruence on $\Pi_{i \in I}^{*} S_{i}$ generated by all pairs $\left(u_{i}, u_{j}\right), u_{i} \in U_{i}, u_{j} \in U_{j}, i, j, \in I$. A replacement of an occurrence of $u_{i}$ in a syllable of $x \in \prod_{i \in I}^{*} S_{i}$ by $u_{j}$ is called a step, and following [5] we distinguish four types of steps (a step is indicated by an arrow):

$\left.* s_{i_{m}}\right)$,

(1) S-steps: $s_{i_{1}} * \cdots * u_{i_{k}} * \cdots * s_{i_{m}} \rightarrow\left(s_{i_{1}} * \cdots\right) * u_{j_{l}} *\left(\cdots * s_{i_{m}}\right)$,

(2) M-steps: $s_{i_{1}} * \cdots * s_{i_{k}} u_{i_{k}} t_{i_{k}} * \cdots * s_{i_{m}} \rightarrow s_{i_{1}} * \cdots * s_{i_{k}} * u_{j_{l}} * t_{i_{k}}$

$* \cdots * s_{i_{m}}$,

(3) $E_{r}$-steps: $s_{i_{1}} * \cdots * s_{i_{k}} u_{i_{k}} * \cdots * s_{i_{m}} \rightarrow\left(s_{i_{1}} * \cdots * s_{i_{k}}\right) * u_{i_{l}} *(\cdots$ $\left.* s_{i_{m}}\right)$.

(4) $E_{l}$-steps: $s_{i_{1}} * \cdots * u_{i_{k}} t_{i_{k}} * \cdots * s_{i_{m}} \rightarrow\left(s_{i_{1}} * \cdots\right) * u_{j_{l}} *\left(t_{i_{k}} * \cdots\right.$

Note that an $M$-step always introduces a new syllable, while an $S$-step or an $E$-step may or may not introduce a new syllable according to the context of $u_{i_{k}}$.

We shall use the notation $x \Rightarrow y$ if there is a succession of steps (also called a transition) $x \rightarrow x_{1} \rightarrow \cdots \rightarrow x_{n-1} \rightarrow y$ linking $x, y \in \Pi_{i \in I}^{*} S_{i}$. Thus, we have $x \rho y$ if and only if there is a transition $\tau: x \Rightarrow y$. Two transitions $\tau_{1}$ and $\tau_{2}$ are called equivalent if they both link the same elements of $\Pi_{i \in I}^{*} S_{i}$. In terms of transitions, condition (iii) of Theorem 1.1 is obviously equivalent to the following:

(iv) (a) If there is a transition $s_{i}^{(1)} \Rightarrow s_{i}^{(2)}$, then $s_{i}^{(1)}=s_{i}^{(2)}$.

(b) If there is a transition $s_{i} \Rightarrow s_{j}(i \neq j)$ then there exists $u \in U$ such that $s_{i}=u_{i}$ and $s_{j}=u_{j}$.

We shall refer to (iv) as "condition (iv) of Theorem 1.1".

For further simplification, whenever there is no ambiguity, we suppress the symbol * in denoting products in $\Pi_{i \in I}^{*} S_{i}$, thus writing elements in the form $s_{i_{1}} s_{i_{2}} \cdots s_{i_{m}}$.

As an illustration of the concepts introduced, we sketch the proofs of some sufficient conditions, related to the conditions in Theorems $1.2,1.3,1.4$, for a semigroup amalgam to be embeddable.

Recall that a subsemigroup $U$ of a semigroup $S$ is said to be left [resp. right] unitary if for all $u \in U, s \in S, u s \in U$ implies $s \in U$ [resp. $s u \in U$ implies $s \in U$ ]. $U$ is said to be unitary if it is both left and right unitary.

Proposition 2.1 (SEe [5, P. 522]). If $U_{i}=h_{i}(U)$ is unitary in $S_{i}$ for every $i \in I$, then the semigroup amalgam $\left[S_{i} ; U ; h_{i}\right]_{i \in I}$ is embeddable.

Proof. The idea is to show that a transition $\tau: x \Rightarrow y$ from $x$ to $y \in$ $\Pi_{i \in I}^{*} S_{i}$ is equivalent to a transition $\tau^{\prime}: x \Rightarrow y$ where all possible $S$-steps occurring in $\tau^{\prime}$ are performed at the beginning. For this purpose one shows that a succession of two steps $M-S$ or $E_{r} S$ or $E_{l}-S$ can be replaced by a transition without $S$-steps or with $S$-steps performed at the beginning. This can always be done without any assumption on $U_{i}$ (see e.g. [5, Lemma 3.10]) except in the following configurations: 
(a)

$$
\begin{gathered}
\cdots s_{i}^{(1)} s_{j} \cdots=\cdots s_{i}^{(2)} u_{i}^{(1)} s_{j} \cdots \stackrel{E_{r}}{\rightarrow} \cdots s_{i}^{(2)} u_{j}^{(1)} s_{j} \cdots \\
\cdots s_{i}^{(2)} u_{j}^{(1)} s_{j} \cdots=\cdots s_{i}^{(2)} u_{j}^{(2)} \cdots \stackrel{s}{\rightarrow} \cdots s_{i}^{(2)} u_{k}^{(2)} \cdots
\end{gathered}
$$

Then from $u_{j}^{(1)} s_{j}=u_{j}^{(2)}$ we deduce that $s_{j}=u_{j}^{(3)}$, so that (a) can be replaced by

$$
\cdots s_{i}^{(2)} u_{j}^{(3)} \ldots \stackrel{s}{\rightarrow} \cdots s_{i}^{(1)} u_{i}^{(3)} \ldots
$$

$\left(\mathrm{a}^{\prime}\right) \cdots s_{i}^{(1)} u_{i}^{(3)} \cdots=\cdots s_{i}^{(2)} u_{i}^{(1)} u_{i}^{(3)} \cdots=\cdots s_{i}^{(2)} u_{i}^{(2)}$

$$
\ldots \stackrel{E_{r}}{\rightarrow} \cdots s_{i}^{(2)} u_{k}^{(2)} \cdots .
$$

There is also a dual configuration $E_{l} S$ that can be replaced similarly by $S-E_{l}$. Replacing a transition $\tau: s_{i}^{(1)} \Rightarrow s_{i}^{(2)}$ by an equivalent transition $\tau^{\prime}$ defined as above and taking in account the fact that an $E$-step or an $M$-step does not diminish the syllable length, one gets $s_{i}^{(1)}=s_{i}^{(2)}$. One shows similarly that $s_{i} \Rightarrow s_{j}$ yields $s_{i}=$ $u_{i}, s_{j}=u_{j}$ for some $u \in U$. A detailed proof is given in [5, Lemma 3.9]. Condition (iv) of Theorem 1.1 is satisfied. Therefore the amalgam is embeddable.

Theorem 1.4, concerning the amalgamation of an ideal, can be proved almost exactly along the same lines as Proposition 2.1.

Proof of THEOREM 1.4. The condition of the theorem is clearly necessary. To show sufficiency, consider an $E_{r}$-step followed by an $S$-step in the configuration (a). Since $U_{i}$ and $U_{j}$ are ideals of $S_{i}$ and $S_{j}$ respectively we have $s_{i}^{(2)} u_{i}^{(1)}=$ $u_{i}^{(3)}$ and $u_{j}^{(1)} s_{j}=u_{j}^{(2)}$. It follows $h_{j}^{-1}\left(u_{j}^{(3)} s_{j}\right)=h_{i}^{-1}\left(s_{i}^{(2)} u_{i}^{(2)}\right)$ and (a) can be replaced by:

$$
\begin{aligned}
\cdots s_{i}^{(1)} s_{j} \cdots=\cdots u_{i}^{(3)} s_{j} \cdots & \stackrel{s}{\rightarrow} \cdots u_{j}^{(3)} s_{j} \cdots \\
& \stackrel{S \text { or } E_{r}}{\longrightarrow} \cdots s_{i}^{(2)} u_{i}^{(2)} \cdots \stackrel{E_{r}}{\rightarrow} s_{i}^{(2)} u_{k}^{(2)} \cdots .
\end{aligned}
$$

The rest of the proof is identical to the proof of Proposition 2.1.

Finally, we proceed to show that the embedding of an amalgam is possible under slightly more general conditions than those of Theorem 1.3. We say that a subsemigroup $U$ of a semigroup $S$ is strongly left unitary if

(1) $U$ is left unitary in $S$ ( $u s \in U, u \in U$ imply $s \in U$ ),

(2) for every $u, v \in U, s, t \in S, u s=v t$ implies that there exist $u^{\prime}, v^{\prime} \in U$, $p \in S$ such that $s=u^{\prime} p, t=v^{\prime} p, u u^{\prime}=v v^{\prime}$.

Note that if, as in Theorem 1.3, there exists a subset $P$ of $S$ such that the mapping $(u, p) \rightarrow u p$ from $U \times P$ into $S$ is a bijection, then $S$ is strongly left unitary. We shall prove that if $\left[S_{i} ; U ; h_{i}\right]_{i \in I}$ is a monoid amalgam such that $h_{i}(U)$ is strongly left unitary in $S_{i}$ for every $i \in I$, then $\left[S_{i} ; U ; h_{i}\right]_{i \in I}$ is embeddable. We need the following notion, which will play a key role in the next section.

Definition 2.2. Let $\left[S_{i} ; U ; h_{i}\right]_{i \in I}$ be a semigroup amalgam. We say that 
two consecutive steps (i.e. replacement of occurrences of $u_{i}=h_{i}(u)$ by $u_{j}=h_{j}(u)$ ) in $\prod_{i \in I}^{*} S_{i}$ are connected if the following conditions are satisfied:

(a) In case the first step introduces a new syllable, then the second step is an $E_{r^{-}}$or $E_{l}$ - or $M$-step and affects the syllable just introduced.

(b) In case the first step does not introduce a new syllable but transfers a $u_{i}$ from a syllable $s_{i}$ to a syllable $s_{j}$, then the second step affects the modified $s_{j}$.

A transition $\tau: x \Rightarrow y$ is called connected if any two consecutive steps of $\tau$ are connected.

LEMma 2.3. Let $\left[S_{i} ; U ; h_{i}\right]_{i \in I}$ be a semigroup amalgam. Suppose $h_{i}(U)$ is strongly left unitary in $S_{i}$ for every $i \in I$. Then connected transitions consisting of two steps of the form $E_{r}-E_{l}, M-E_{l}$ or $E_{r}-S, M-S$ are equivalent to transitions where the $E_{l}$-steps and the $S$-steps are performed first, or not at all.

Proof. Case $E_{r}-E_{l}$. If $E_{r}$ introduces a new syllable we have

$$
\begin{aligned}
\cdots s_{i} \cdots & =\cdots s_{i}^{\prime} u_{i} \cdots \stackrel{E_{r}}{\rightarrow} \cdots s_{i}^{\prime} u_{j} \cdots \\
& =\cdots s_{i}^{\prime} u_{j}^{\prime} s_{j} \cdots \stackrel{E_{l}}{\rightarrow} \cdots s_{i}^{\prime} u_{i}^{\prime} s_{j} \cdots \text { or } \cdots s_{i}^{\prime} u_{k}^{\prime} s_{j} \cdots .
\end{aligned}
$$

From $u_{j}=u_{j}^{\prime} s_{j}$ we deduce that $s_{j} \in U_{j}$ and $s_{j}=u_{j}^{\prime \prime}$. Thus we have the equivalent transitions:

$$
\begin{aligned}
& \cdots s_{i} \cdots=\cdots s_{i}^{\prime} u_{i}^{\prime} u_{i}^{\prime \prime} \cdots \stackrel{E_{r}}{\rightarrow} \cdots s_{i}^{\prime} u_{i}^{\prime} u_{j}^{\prime \prime} \cdots=\cdots s_{i}^{\prime} u_{i}^{\prime} s_{j} \cdots \text { or } \\
& \cdots s_{i} \cdots=\cdots s_{i}^{\prime} u_{i}^{\prime} u_{i}^{\prime \prime} \cdots \stackrel{E_{r}}{\rightarrow} \cdots s_{i}^{\prime} u_{i}^{\prime} u_{j}^{\prime \prime} \cdots \stackrel{E_{r}}{\rightarrow} \cdots s_{i}^{\prime} u_{k}^{\prime} u_{j}^{\prime \prime} \cdots=\cdots s_{i}^{\prime} u_{k}^{\prime} s_{j} \cdots .
\end{aligned}
$$

If $E_{r}$ does not introduce a new syllable we have

$$
\begin{aligned}
\cdots s_{i} s_{j} \cdots & =\cdots s_{i}^{\prime} u_{i} s_{j} \cdots \stackrel{E_{r}}{\rightarrow} \cdots s_{i}^{\prime} u_{j} s_{j} \cdots \\
& =\cdots s_{i}^{\prime} u_{j}^{\prime} s_{j}^{\prime} \cdots \stackrel{E_{l}}{\rightarrow} \cdots s_{i}^{\prime} u_{i}^{\prime} s_{j}^{\prime} \cdots \text { or } \cdots s_{i}^{\prime} u_{k}^{\prime} s_{j}^{\prime} \cdots
\end{aligned}
$$

But $u_{j} s_{j}=u_{j}^{\prime} s_{j}^{\prime}$ implies $s_{j}=v_{j} p_{j}, s_{j}^{\prime}=v_{j}^{\prime} p_{j}, u_{j} v_{j}=u_{j}^{\prime} v_{j}^{\prime}$ for some $v_{j}, v_{j}^{\prime} \in U_{j}, p_{j} \in S_{j}$. Thus we obtain equivalent transitions as follows:

$$
\begin{aligned}
\cdots s_{i} s_{j} \cdots & =\cdots s_{i}^{\prime} u_{i} v_{j} p_{j} \cdots \stackrel{E_{l}}{\rightarrow} \cdots s_{i}^{\prime} u_{i} v_{i} p_{j} \cdots \\
& =\cdots s_{i}^{\prime} u_{i}^{\prime} v_{i}^{\prime} p_{j} \cdots \stackrel{E_{r}}{\rightarrow} \cdots s_{i}^{\prime} u_{i}^{\prime} v_{j}^{\prime} p_{j} \cdots=\cdots s_{i}^{\prime} u_{i}^{\prime} s_{j}^{\prime} \cdots \text { or } \\
\cdots s_{i}^{\prime} u_{i} v_{j} p_{j} & \cdots \stackrel{E_{l}-S}{\longrightarrow} \cdots s_{i}^{\prime} u_{k} v_{k} p_{j} \cdots \\
& =\cdots s_{i}^{\prime} u_{k}^{\prime} v_{k}^{\prime} p_{j} \cdots \stackrel{E_{r}}{\rightarrow} \cdots s_{i}^{\prime} u_{k}^{\prime} v_{j}^{\prime} p_{j} \cdots=\cdots s_{i}^{\prime} u_{k}^{\prime} s_{j}^{\prime} \cdots \cdots
\end{aligned}
$$

The other cases $M-E_{l}, E_{r}-S, M-S$ are treated similarly; they use only the left unitary condition or no condition at all for $M-S$.

As a consequence of Lemma 2.3, it is possible either to suppress or to shift $S$-steps or $E_{l}$-steps forward in a transition, provided they follow an $E_{r}$-step or an 
$M$-step. This is proved as in Lemma 3.10 of [5] the most critical cases being when the following $M-S$ configurations occur:

(1) $\cdots s_{i} u_{i} u_{i}^{\prime} \cdots \stackrel{M}{\rightarrow} s_{i} u_{j} u_{i}^{\prime} \cdots \stackrel{s}{\rightarrow} \cdots s_{i} u_{j} u_{k}^{\prime} \cdots$,

They can be replaced respectively by:

(1') $\cdots s_{i} u_{i} u_{i}^{\prime} \cdots \frac{E_{r}}{E_{r}} \cdots s_{i} u_{j} u_{j}^{\prime} \cdots \frac{E_{r}}{E_{r}} \cdots s_{i} u_{j} u_{k}^{\prime} \cdots$,

$\left(2^{\prime}\right) \cdots u_{i}^{\prime} u_{i} t_{i} \cdots \stackrel{E_{l}}{\longrightarrow} \cdots u_{k}^{\prime} u_{k} t_{i} \cdots \stackrel{E_{r}}{\longrightarrow} \cdots u_{k}^{\prime} u_{j} t_{i} \cdots$.

Proposition 2.4. Let $\left[S_{i} ; U ; h_{i}\right]_{i \in I}$ be a monoid amalgam (i.e. $S_{i}$ and $U$ are monoids and $h_{i}(e)=e_{i}$ for every $\left.i \in I\right)$. Suppose $h_{i}(U)$ is strongly left unitary in $S_{i}$ for every $i \in I$. Then the amalgam is embeddable.

Proof. Let $\tau: x \Rightarrow y$ be a transition from $x$ to $y$, with $x, y \in \prod_{i \in I}^{*} S_{i}$. Introducing eventually additional steps in $\tau$, where identity elements are shifted from one syllable to another, we may assume that all two consecutive steps in $\tau$ form connected transitions. It follows from Lemma 2.3 that $\tau$ is equivalent to a transition $\tau^{\prime}$ where all the $S$-steps and the $E_{l}$-steps are performed at the beginning of $\tau^{\prime}$. We will show that $\tau^{\prime}$ is equivalent to a transition $\tau^{\prime \prime}$ with the following structure: $\tau^{\prime \prime}$ begins with a succession of $E_{l}$-steps, followed by a succession of $S$ steps, itself followed by a succession of steps that are not $E_{l}$ or $S$-steps. To establish this, it is enough to show that an $S$-step followed by an $E_{l}$-step can be replaced by a sequence of the form $E_{l}-E_{l} \cdots \cdots-E_{l}-S \cdot S \cdot \cdots-S$. The cases when an $S$-step followed by an $E_{l}$-step are not immediately interchangeable are
(1) $\cdots s_{k} u_{i} s_{l} \cdots \stackrel{s}{\rightarrow} \cdots s_{k} u_{j} s_{l} \cdots=\cdots s_{k} u_{j}^{\prime} s_{j} s_{l} \cdots \stackrel{E_{l}}{\rightarrow} \cdots s_{k} u_{m}^{\prime} s_{j} s_{l}$
$\cdots$,
(2) $\cdots s_{k} u_{i} s_{l} \cdots \stackrel{s}{\rightarrow} \cdots s_{k} u_{k} s_{l} \cdots=\cdots u_{k}^{\prime} s_{k}^{\prime} s_{l} \cdots \stackrel{E_{l}}{\rightarrow} \cdots u_{m}^{\prime} s_{k}^{\prime} s_{l}$
$\cdots$,
(3) $\cdots s_{k} u_{i} s_{l} \cdots \stackrel{s}{\rightarrow} \cdots s_{k} u_{l} s_{l} \cdots=\cdots s_{k} u_{l}^{\prime} s_{l}^{\prime} \cdots \stackrel{E_{l}}{\rightarrow} \cdots s_{k} u_{m}^{\prime} s_{l}^{\prime}$

In case (1), from $u_{j}=u_{j}^{\prime} s_{j}$ we deduce $s_{j}=u_{j}^{\prime \prime}$ and (1) is equivalent to

(1') $\cdots s_{k} u_{i} s_{l} \cdots=\cdots s_{k} u_{i}^{\prime} u_{i}^{\prime \prime} s_{l} \cdots E_{l} \cdots s_{k} u_{m}^{\prime} u_{i}^{\prime \prime} s_{l} \cdots \stackrel{s}{\rightarrow} \cdots$ $s_{k} u_{m}^{\prime} u_{j}^{\prime \prime} s_{l} \cdots=\cdots s_{k} u_{m}^{\prime} s_{j} s_{l} \cdots$.

In case (2), we use the fact that we have a monoid amalgam; (2) is equivalent to

(2') $\cdots s_{s} u_{i} s_{l} \cdots=\cdots s_{k} u_{i} e_{i} s_{l} \cdots \stackrel{E_{l}}{\longrightarrow} \cdots s_{k} u_{k} e_{i} s_{l} \cdots \stackrel{E_{l}}{\longrightarrow} \cdots$ $u_{m}^{\prime} s_{k}^{\prime} e_{i} s_{l} \cdots \stackrel{s}{\rightarrow} \cdots u_{m}^{\prime} s_{k}^{\prime} e_{k} s_{l} \cdots=\cdots u_{m}^{\prime} s_{k}^{\prime} s_{l} \cdots$.

In case (3), from $u_{l} s_{l}=u_{l}^{\prime} s_{l}^{\prime}$ we deduce $s_{l}=v_{l} p_{l}, s_{l}^{\prime}=v_{l}^{\prime} p_{l}, u_{l} v_{l}=u_{l}^{\prime} v_{l}^{\prime}$ for some $p_{l} \in S_{l}$, and $v_{l}, v^{\prime} \in U_{l}$. It follows that (3) is equivalent to

(3') $\cdots s_{k} u_{i} s_{l} \cdots=\cdots s_{k} u_{i} v_{l} p_{l} \cdots E_{l} \cdots s_{k} u_{i} v_{i} p_{l} \cdots=\cdots$ $s_{k} u_{i}^{\prime} v_{i}^{\prime} p_{l} \cdots \stackrel{E_{l}}{\longrightarrow} \cdots s_{k} u_{m}^{\prime} v_{i}^{\prime} p_{l} \cdots \stackrel{s^{i}}{\rightarrow} \cdots s_{k} u_{m}^{\prime} v_{l}^{\prime} p_{l} \cdots \stackrel{k_{i}}{=} \cdots s_{k} u_{m}^{\prime} s_{l}^{\prime} \cdots$. 
Suppose now that $\tau$ is a transition from $s_{i}^{(1)} \in S_{i}$ to $s_{j}^{(2)} \in S_{j}$ (eventually $i=j$ ). Then $\tau$ is equivalent to a transition $\tau^{\prime \prime}$ with the properties indicated above. Since $E_{r}$-steps or $M$-steps do not diminish the syllable length, it follows that when used in $\tau^{\prime \prime}$ they must be applied to one-syllable words and thus increase the syllable length. The transition $\tau^{\prime \prime}$ linking two one-syllable words, it follows that $\tau^{\prime \prime}$ consists in a succession of $E_{l}$-steps followed by $S$-steps. Using the left unitary condition, a simple induction argument shows that a succession of $E_{\text {-steps transforms }}$ $s_{i}^{(1)}$ into $u_{i_{1}}^{(1)} u_{i_{2}}^{(2)} \cdots u_{i_{n}}^{(n)} s_{i}^{(3)}$ where $s_{i}^{(1)}=u_{i}^{(1)} u_{i}^{(2)} \cdots u_{i}^{(n)} s_{i}^{(3)}$. In case $j \neq i$, the $S$-steps in $\tau^{\prime \prime}$ must affect $s_{i}^{(3)}$. Thus $s_{i}^{(3)}=u_{i}^{(n+1)}$ and $s_{j}^{(1)}=u_{j}^{(1)} u_{j}^{(2)}$ $\cdots u_{j}^{(n)} u_{j}^{(n+1)}$, showing that $s_{i}^{(1)}=u_{i}$ and $s_{j}^{(2)}=u_{j}$. In case $j=i$, the $S$-steps in $\tau^{\prime \prime}$ may or not affect $s_{i}^{(3)}$. If they do, then $s_{i}^{(1)}=u_{i}=s_{i}^{(2)}$ with $u_{i}=u_{i}^{(1)} u_{i}^{(2)}$ $\cdots u_{i}^{(n)} s_{i}^{(3)}$ and if they do not $s_{i}^{(1)}=u_{i} s_{i}^{(3)}=s_{i}^{(2)}$ with $u_{i}=u_{i}^{(1)} u_{i}^{(2)} \cdots u_{i}^{(n)}$. Condition (iv) of Theorem 1.1 is satisfied, proving that the amalgam is embeddable.

\section{A necessary and sufficient condition for the embedding of semigroup} amalgams. Let $\left[S_{i} ; U ; h_{i}\right]_{i \in I}$ be a semigroup amalgam, and let

$$
\tau: x=x_{0} \rightarrow x_{1} \rightarrow x_{2} \rightarrow \cdots \rightarrow x_{n}=y
$$

be a connected transition (see Definition 2.2) from $x$ to $y$, with $x=x_{0}, x_{1}, \cdots$, $x_{n}=y \in \Pi_{i \in I}^{*} S_{i}$. Given any two consecutive steps $x_{i-1} \rightarrow x_{i} \rightarrow x_{i+1}(0<i<n)$ in $\tau$, there is a syllable of $x_{i}$ which admits two factorizations, the first one reflecting the result of the step $x_{i-1} \rightarrow x_{i}$ and the second reflecting the origin of $x_{i} \rightarrow$ $x_{i+1}$. Consider for example, the following connected $E_{r}-E_{l}$ steps $\cdots s_{k} u_{k}^{(1)} s_{l}^{(1)} \ldots \stackrel{E_{r}}{\rightarrow} s_{k} u_{l}^{(1)} s_{l}^{(1)} \ldots=\cdots s_{k} u_{l}^{(2)} s_{l}^{(2)} \ldots \stackrel{E_{l}}{\rightarrow} \cdots s_{k} u_{k}^{(2)} s_{l}^{(2)} \ldots$.

The syllable with index $l$ obtained after $E_{r}$ has the factorization $u_{l}^{(1)} s_{l}^{(1)}$ ("outcome" of $E_{r}$ ) and the factorization $u_{l}^{(2)} s_{l}^{(2)}$ ("input" of $E_{l}$ ).

An equality arising from two connected steps $\left(u_{l}^{(1)} s_{l}^{(1)}=u_{l}^{(2)} s_{l}^{(2)}\right)$ of the transition $\tau$ is called a connecting equality of $\tau$.

Definition 3.1. Let $I$ be a set, and let $s_{i}^{(k)}, u_{i}^{(k)}, i \in I, k \in \mathrm{N}$, be sequences of letters forming an alphabet $A$. A system $\Sigma$ of equations is a set of (unordered) pairs of elements of the free semigroup over $A$. If $\left(w, w^{\prime}\right) \in \Sigma$ we write $w=w^{\prime}$. A system $\Sigma$ is called a coherent system of equations, if there exist a semigroup amalgam $\left[S_{i} ; U ; h_{i}\right]_{i \in I}$ and a connected transition $\tau$ in $\Pi_{i \in I}^{*} S_{i}$ such that the equations of $\Sigma$ are the connecting equalities of $\tau$.

EXAMPLE. Let $\tau$ be the connected transition

$$
\begin{aligned}
s_{2}^{(1)} s_{1}^{(1)} u_{3}^{(1)} \rightarrow s_{2}^{(1)} s_{1}^{(1)} u_{1}^{(1)}=s_{2}^{(1)} u_{1}^{(2)} s_{1}^{(2)} & \rightarrow s_{2}^{(1)} u_{2}^{(2)} s_{1}^{(2)}=u_{2}^{(3)} s_{1}^{(2)} \\
& \rightarrow u_{3}^{(3)} s_{1}^{(2)}=s_{3}^{(1)} u_{3}^{(4)} s_{3}^{(2)} s_{1}^{(2)} .
\end{aligned}
$$


The coherent system corresponding to $\tau$ is

$$
s_{1}^{(1)} u_{1}^{(1)}=u_{1}^{(2)} s_{1}^{(2)}, \quad s_{2}^{(1)} u_{2}^{(2)}=u_{2}^{(3)}, \quad u_{3}^{(3)}=s_{3}^{(1)} u_{3}^{(4)} s_{3}^{(2)} .
$$

In order to define a coherent system of equations independently from any reference to a connected transition, note that for any equation $E$ in a coherent system, the left side and the right side of $E$ are words over $A$ of the type $u_{i}^{(l)}, u_{i}^{(l)} s_{i}^{(m)}$, $s_{i}^{(k)} u_{i}^{(l)}, s_{i}^{(k)} u_{i}^{(l)} s_{i}^{(m)}$ with the same index for each side of $E$ and with the exception that $E$ is not $u_{i}^{\left(l_{1}\right)}=u_{i}^{\left(l_{2}\right)}$. Thus there are 15 possible types of equations. If the right side of $E$ is $u_{i}^{(l)}$ we say that the $u$-variable appears freely in $E$. If the right side of $E$ is $u_{i}^{(l)} s_{i}^{(m)}$ [resp. $s_{i}^{(k)} u_{i}^{(l)}$ ] we say that the $u$-variable is blocked on the right [resp. left] in $E$ by $s_{i}^{(m)}$ [resp. $s_{i}^{(k)}$ ]. Finally if the right side of $E$ is $s_{i}^{(k)} u_{i}^{(l)} s_{i}^{(m)}$ we say that the $u$-variable is blocked in $E$ by $s_{i}^{(k)}$ and $s_{i}^{(m)}$. This terminology is used in our next inductive construction of a coherent system.

Construction 3.2. Let $I$ be a set, $s_{i}^{(k)}, u_{i}^{(k)}(i \in I, k \in \mathrm{N})$ sequences of letters forming an alphabet $A$. We construct a finite system of equations $E_{1}, E_{2}$, $\cdots, E_{r}$ over $A$ as follows:

(1) For every $n(1 \leqslant n \leqslant r)$ the letters in $E_{n}$ have all the same index $i_{n}$ and $i_{n} \neq i_{n+1}$ for $1 \leqslant n<r$. Furthermore $E_{n}$ is not $u_{i_{n}}^{(k)}=u_{i_{n}}^{(l)}$ for all $n, 1 \leqslant$ $n \leqslant r, k, l \in \mathrm{N}$.

(2) For every $i \in I$, the letters $u_{i}^{(k)}$ appearing in all equations $E_{n}$ such that $i_{n}=i$ have distinct superscripts, and the letters $s_{i}^{(k)}$ appearing in a given equation also have distinct superscripts.

(3) $E_{1}$ is any one of the 15 possible types of equations indicated above.

(4) Suppose $E_{1}, E_{2}, \cdots, E_{n-1}(n>1)$ have been defined. Then the right side of $E_{n}$ can be $u_{i_{n}}^{(l)}, u_{i_{n}}^{(l)} s_{i_{n}}^{(m)}, s_{i_{n}}^{(k)} u_{i_{n}}^{(l)}$ or $s_{i_{n}}^{(k)} u_{i_{n}}^{(l)} s_{i_{n}}^{(m)}$, for some $k, l, m \in \mathrm{N}$ except for the case outruled by (1).

The restrictions for the left side of $E_{n}$ depend first on the nature of the right side of $E_{n-1}$. They are indicated on the following table:

\begin{tabular}{l|c|c|c|c} 
Right side of $E_{n-1}$ & $u_{i_{n-1}}^{(l)}$ & $u_{i_{n-1}}^{(l)} s_{i_{n-1}}^{(m)}$ & $s_{i_{n-1}}^{(k)} u_{i_{n-1}}^{(l)}$ & $s_{i_{n-1}}^{(k)} u_{i_{n-1}}^{(l)} s_{i_{n-1}}^{(m)}$ \\
\hline $\begin{array}{l}\text { Possible left sides } \\
\text { of } E_{n}\end{array}$ & $u_{i_{n}}^{(l)}$ & $u_{i_{n}}^{(l)}$ & $u_{i_{n}}^{(l)}$ & $u_{i_{n}}^{(l)}$ \\
& $s_{i_{n}}^{(k)} u_{i_{n}}^{(l)}$ & $s_{i_{n}}^{(k)} u_{i_{n}}^{(l)}$ & $u_{i_{n}}^{(l)} s_{i_{n}}^{(m)}$ & \\
& $u_{i_{n}}^{(l)} s_{i_{n}}^{(m)}$ & & & \\
& & & \\
$s_{i_{n}}^{(k)} u_{i_{n}}^{(l)} s_{i_{n}}^{(m)}$ & & &
\end{tabular}


Further restrictions on the contexts of $u_{i_{n}}^{(l)}$ are as follows:

(a) If the $u$-variable appears freely in $E_{k}$ for every $k<n$, there are no restrictions on the context of $u_{i_{n}}^{(l)}$.

(b) If, for every $k<n$, the $u$-variable is blocked on the left [resp. right] by $s_{i_{k}}^{(k)}$ [resp. $s_{i_{k}}^{(m)}$ ] and $s_{i_{k}}^{(k)}$ [resp. $s_{i_{k}}^{(m)}$ ] appears on the left side of $E_{k+1}, \cdots$, $E_{n-1}$, there is no restriction on the left [resp. right] context of $u_{i_{n}}^{(l)}$.

(c) If the $u$-variable does not appear freely in $E_{k}$ for every $k<n$, let $p$ [resp. $q$ ] be the largest integer $\langle n-1$ such that the $u$-variable is blocked on the left by $s_{i_{p}}^{(k)}$ [resp. on the right by $s_{i_{q}}^{(m)}$ ] and such that $s_{i_{p}}^{(k)}$ [resp. $s_{i q}^{(m)}$ ] does not appear on the left side of any of the equations $E_{p+1}, \cdots,{ }^{q}, E_{n-1}$ [resp. $E_{q+1}, \cdots$, $E_{n-1}$ ]. If $i_{n} \neq i_{p}$ [resp. $i_{n} \neq i_{q}$ ], then $u_{i_{n}}$ has no left [resp. right] context. If $i_{n}=i_{p}$ [resp. $i_{n}=i_{q}$ ], then the left [resp right] context of $u_{i_{n}}$ is $s_{i_{p}}^{(k)}$ [resp. $s_{i_{q}}^{(m)}$ ].

Justification. Suppose that the equations of a system $\Sigma$ are the connecting equalities of a transition $\tau$ in $\Pi_{i \in I}^{*} S_{i}$ where $\left[S_{i} ; U ; h_{i}\right]_{i \in I}$ is a semigroup amalgam. There is no difficulty verifying (1), (2), (3) and the restrictions concerning the type of the right and left side of $E_{n}$. To verify (4)(a) assume that the $u$-variable appears freely in $E_{k}(k<n)$. Then none of the first $n$ steps of $\tau$ introduces an $s$-variable. Consequently the contexts of $u_{i_{n}}^{(l)}$ do not depend on the equations $E_{1}$, $E_{2}, \cdots, E_{n-1}$. Similarly if the $u$-variables are blocked for example on the left, by variables $s_{i_{k}}^{(k)}$ appearing on the left side of a following equation, the left context of $u_{i_{n}}^{(l)}$ does not depend on $E_{1}, E_{2}, \cdots, E_{n-1}$. Finally s-variables blocking $u$-variables and not appearing on the left side of a following equation are $s$-variables introduced by the successive steps of $\tau$ and not suppressed by any of them. They are present in the successive elements of $\Pi_{i \in I}^{*} S_{i}$ involved in $\tau$, in their order of appearance. Consequently the left context of $u_{i_{n}}^{(l)}$ (if any) must be $s_{i_{p}}^{(k)}$ as defined (4)(b).

Conversely, let $\Sigma$ be a system of equations obeying conditions (1) to (4). For a fixed $i \in I$ let $S_{i}$ be the semigroup generated by all $s_{i}^{(k)}, u_{i}^{(l)}$ where $s_{i}^{(k)}$ belongs to the set of all $s$-variables with index $i$ appearing in $\Sigma$ and $u_{i}^{(l)}$ belongs to the set of all $u$-variables such that $u_{j}^{(l)}$ appears in $\Sigma$ for some $j \in I$. Suppose that the presentation relations for $S_{i}$ are all the equations of $\Sigma$ involving $s$-variables and $u$-variables with index $i$. By condition (2) the subsemigroup of $S_{i}$ generated by all $u_{i}^{(l)}$ is free. Thus, if $U$ is the free semigroup on the set $\left\{u^{(l)}:\right.$ there exists $j \in I$ that $u_{j}^{(l)}$ appears in $\Sigma$ \}, then there is a 1-1 homomorphism $h_{i}: U \rightarrow S_{i}$ for every $i \in I$ and $\left[S_{i} ; U ; h_{i}\right]_{i \in I}$ is a semigroup amalgam. We show that there is a transition $\tau$ in $\Pi_{i \in I}^{*} S_{i}$ admitting the equations $E_{1}, \cdots, E_{r}$ of $\Sigma$ as connecting equalities. We shall say that an $s$-variable $s_{i}^{(m)}$ is at the origin [resp. the extremity] of $\Sigma$ if $s_{i}^{(m)}$ appears on the left [resp. right] side of an equation $E_{k}(1 \leqslant k \leqslant r)$ but 
does not appear on the right [resp. left] side of an equation $E_{1}$ for $l<k$ [resp. for $l>k$ ]. Let $x=s_{j_{r}}^{\left(l_{r}\right)} \cdots s_{j_{2}}^{\left(l_{2}\right)} s_{j_{1}}^{\left(l_{1}\right)} u_{n}^{(1)} s_{k_{1}}^{\left(m_{1}\right)} s_{k_{2}}^{\left(m_{2}\right)} \cdots s_{k_{t}}^{\left(m_{t}\right)}$ be the element of $\Pi_{i \in I}^{*} S_{i}$ obtained by the following process: $u_{i_{1}}^{(1)}$ is the $u$-variable in the left side of $E_{1}$ and $n \neq i_{1} ; s_{j_{1}}^{\left(l_{1}\right)}, s_{j_{2}}^{\left(l_{2}\right)}, \cdots, s_{j_{r}}^{\left(l_{r}\right)}$ are $s$-variables at the origin of $\Sigma$ that are left contexts of $u$-variables, written from right to left in their order of appearance; similarly $s_{k_{1}}^{\left(m_{1}\right)}, s_{k_{2}}^{\left(m_{2}\right)}, \cdots, s_{k_{t}}^{\left(m_{t}\right)}$ are right contexts of $u$-variables at the origin of $\Sigma$ written from left to right in their order of appearance. Dually define $y \in \Pi_{i \in I}^{*} S_{i}$ using as $u$-variable the variable appearing on the right side of $E_{r}$, and using $s$-variables at the extremity of $\Sigma$. Then by conditions (3), (4) it is possible to construct inductively a connected transition $\tau: x \Rightarrow y$ with the equations of $\Sigma$ as connecting equalities. The details involve only notational complications and thus are omitted.

The next definition uses the notion of an s-variable at the origin, or the extremity of a connected system of equations $\Sigma$, as defined above in the justification of Construction 3.2.

Definition 3.2. Let $\Sigma$ be a connected system of equation $E_{1}, E_{2}, \cdots$, $E_{r} . \Sigma$ is called a balanced system if

(1) the left side of $E_{1}$ is $u_{i_{1}}^{(1)}$ and the right side of $E_{r}$ is $u_{i_{r}}^{(n)}$,

(2) there is at most one s-variable $s_{i}^{(l)}$ [resp. $s_{j}^{\left(l^{\prime}\right)}$ ] at the origin [resp. extremity] of $\Sigma$ which is a left context, and there is at most one $s$-variable $s_{i}^{(m)}$ [resp. $s_{j}^{\left(m^{\prime}\right)}$ ] at the origin [resp. extremity] of $\Sigma$ which is a right context.

If $i=j$ we say that $\Sigma$ is a balanced system of type $\mathrm{I}$, and if $i \neq j \Sigma$ is called a balanced system of type II. Given a balanced system of type I we call the equation $s_{i}^{(l)} u_{i}^{(1)} s_{i}^{(m)}=s_{i}^{\left(l^{\prime}\right)} u_{i}^{(n)} s_{i}^{\left(m^{\prime}\right)}$ the equation locked to $\Sigma$. Note that the $s$-variables in the locked equation might be present or not. For a balanced system of type II the sentence: "there exists $u_{i}^{(0)}, u_{j}^{(0)}$ such that $s_{i}^{(l)} u_{i}^{(1)} s_{i}^{(m)}=u_{i}^{(0)}$ and $s_{i}^{\left(l^{\prime}\right)} u_{i}^{(n)} s_{i}^{(m)}=u_{j}^{(0)}$ " is called the sentence locked to $\Sigma$.

EXAMPLES. The following are balanced systems with their locked equation or sentence:

$$
\begin{array}{rlrl}
\text { TYPE I } & \text { TYPE II } \\
u_{2}^{(1)} & =u_{2}^{(2)} s_{2}^{(1)} & u_{2}^{(1)} & =s_{2}^{(1)} u_{2}^{(2)} \\
s_{1}^{(1)} u_{1}^{(2)} & =s_{1}^{(2)} u_{1}^{(3)} s_{1}^{(3)} & u_{3}^{(2)} & =u_{3}^{(3)} s_{3}^{(1)} \\
u_{2}^{(3)} & =s_{2}^{(2)} u_{2}^{(4)} & s_{2}^{(1)} u_{2}^{(3)} & =u_{2}^{(4)} s_{2}^{(2)} \\
u_{1}^{(4)} s_{1}^{(3)} & =u_{1}^{(5)} & s_{1}^{(1)} u_{1}^{(4)} & =u_{1}^{(5)} \\
s_{2}^{(2)} u_{2}^{(5)} s_{2}^{(1)} & =u_{2}^{(6)} & u_{2}^{(5)} s_{2}^{(2)} & =u_{2}^{(6)}
\end{array}
$$


Locked equation: $s_{1}^{(1)} u_{1}^{(1)}=s_{1}^{(2)} u_{1}^{(6)}$.

Locked sentence: there exists $u_{1}^{(0)}, u_{3}^{(0)}$ such that $s_{1}^{(1)} u_{1}^{(1)}=u_{1}^{(0)}, u_{3}^{(6)} s_{3}^{(1)}=u_{3}^{(0)}$.

Our main result is:

THEOREM 3.3. A semigroup amalgam $\left[S_{i} ; U ; h_{i}\right]_{i \in I}$ is embeddable if and only if whenever a balanced system of equations $\Sigma$ holds in $S_{i}$ and $U_{i}=h_{i}(U)$ $(i \in I)$, then the equation or the sentence locked to $\Sigma$ also holds in the semigroups $S_{i}(i \in I)$.

By condition (iv) of Theorem 1.1 and Definition 3.2 of a balanced system, the condition of Theorem 3.3 is obviously necessary. That it is also sufficient follows directly from Lemma 3.5 below.

Let $\left[S_{i} ; U ; h_{i}\right]_{i \in I}$ be a semigroup amalgam and let $\sigma$ be a transition in $\Pi_{i \in I}^{*} S_{i}$ of the form $\sigma: x=s_{i_{1}} s_{i_{2}} \cdots s_{i_{k}} \Rightarrow y$. We define the notion of descendant of a given syllable $s_{i_{m}}$ of $x$ as follows. Consider the first step of $\sigma$ modifying the syllable $s_{i_{m}}$. This step might incorporate $s_{i_{m}}$ into a new syllable of one of the forms $a_{i_{m}} s_{i_{m}} b_{i_{m}}, a_{i_{m}} s_{i_{m}}, s_{i_{m}} b_{i_{m}}$ or it might create new syllables after a decomposition of $s_{i_{m}}$ of one the forms $a_{i_{m}}^{(1)} u_{i_{m}}, u_{i_{m}} a_{i_{m}}^{(2)}, a_{i_{m}}^{(1)} u_{i_{m}} a_{i_{m}}^{(2)}$. The new syllables created by the first modifying step of $s_{i_{m}}$ are called the direct descendants of $s_{i_{m}}$. A descendant of $s_{i_{m}}$ is either $s_{i_{m}}$ or a syllable $t$ in $\sigma$ such that there exists a succession of syllables $t_{0}, t_{1}, \cdots, t_{n}$ with $t_{0}=s_{i_{m}}, t_{n}=t$ and $t_{i+1}$ is a direct descendant of $t_{i}$ for $i=0,1, \cdots, n-1$.

In case the first modifying step of $s_{i_{m}}$ is due to a factorization of $s_{i_{m}}$ into $a_{i_{m}}^{(1)} u_{i_{m}}$ or $u_{i_{m}} a_{i_{m}}^{(2)}$ or $a_{i_{m}}^{(1)} u_{i_{m}} a_{i_{m}}^{(2)}$, then $a_{i_{m}}^{(1)}$ and $a_{i_{m}}^{(2)}$ might be modified by subsequent steps of $\sigma$, and this modification might be due to factorizations of $a_{i_{m}}^{(1)}$ or $a_{i_{m}}^{(2)}$. If all the factorizations due to the successive steps of $\sigma$ are reported on $s_{i_{m}}$, then $s_{i_{m}}$ takes one of the forms

$$
\left.s_{i_{m}}=u_{i_{m}} \quad \text { or } \quad s_{i_{m}}=u_{i_{m}}^{(1)} a_{i_{m}}^{(1)} u_{i_{m}}^{(2)} a_{i_{m}}^{(2)} \cdots u_{i_{m}}^{\left({ }^{\prime}\right.}{ }^{\prime}\right) a_{i_{m}}^{\left(n_{m}\right)} u_{i_{m}}^{\left(n_{m}+1\right)}
$$

where some of the $u_{i_{m}}^{(k)}$ 's might be empty symbols. It follows that

$$
\text { (1) } x=s_{i_{1}} s_{i_{2}} \cdots s_{i_{k}}=\prod_{m=1}^{k} u_{i_{m}}^{(1)} a_{i_{m}}^{(1)} u_{i_{m}}^{(2)} a_{i_{m}}^{(2)} \cdots u_{i_{m}}^{\left(n_{m}\right)} a_{i_{m}}^{\left(n_{m}\right)} a_{i_{m}}^{\left(n_{m}+1\right)} \text {. }
$$

We define the descendants of an element $a_{i_{m}}^{(j)}$ as the descendants of the syllable containing $a_{i_{m}}^{(j)}$ when the modification of $a_{i_{m}}^{(j)}$ occurs (by modification of $a_{i_{m}}^{(j)}$ we mean the step $x_{t} \rightarrow x_{t+1}$ of $\sigma$ where $a_{i_{m}}^{(j)}$ appears in a syllable of $x_{t}$ but does not appear in $x_{t+1}$ ).

On the set $A_{x}^{\sigma}=\left\{a_{i_{m}}^{(j)} \mid a_{i_{m}}^{(j)}\right.$ is in the decomposition (1) of $\left.x\right\}$ we define a 
relation $\gamma$ by $a_{i_{m}}^{(j)} \gamma a_{i_{m}}^{\left(j^{\prime}\right)}$, if the modifications of $a_{i_{m}}^{(j)}$ and $a_{i_{m}}^{\left(j^{\prime}\right)}$ occur simultaneously in case both are modified, or if $m=m^{\prime}, j=j^{\prime}$ if $a_{i_{m}}^{(j)}$ is not modified. It is easy to verify that $\gamma$ is an equivalence relation on $A_{x}^{\sigma}$. Furthermore, $a_{i_{m}}^{(j)} \gamma a_{i_{m}}^{\left(j^{\prime}\right)}$ implies $m=m^{\prime}$, because both disappear simultaneously, and thus they are in the same syllable of $x_{t}$, there $x_{t} \rightarrow x_{t+1}$ is their modifying step.

Denoting by $\gamma\left[a_{i_{m}}^{(j)}\right]$ the $\gamma$-class of $a_{i_{m}}^{(j)}$, we define a relation $\leqslant$ on the quotient set $A_{x}^{\sigma} / \gamma$ by

(a) $\gamma\left[a_{i_{m}}^{(j)}\right] \leqslant \gamma\left[a_{i_{i^{\prime}}}^{\left(j^{\prime}\right)}\right]$ if the syllable containing $a_{i_{m}}^{(j)}$ when $a_{i_{m}}^{(j)}$ is modified, is a descendant of $a_{i_{m}}^{\left(j^{\prime}\right)}$,

(b) $\gamma\left[a_{i_{m}}^{(j)}\right] \leqslant \gamma\left[a_{i_{m}}^{\left(j^{\prime}\right)}\right]$ if $a_{i_{m}}^{(j)}$ is unmodified and $a_{i_{m}}^{\left(j^{\prime}\right)}$ is modified or if $a_{i_{m}}^{(j)}$ and $a_{i^{\prime}}^{\left(j^{\prime}\right)}$ are both unmodified and $m=m^{\prime}, j=j^{\prime}$.

The relation $\leqslant$ is reflexive, antisymmetric (since $\gamma$ is an equivalence) and transitive (by transitivity of the descendance relation). Thus it is a partial ordering of $A_{x}^{\sigma} / \gamma$. Note that if $a_{i_{m}}^{(j)}$ is unmodified by $\sigma$, then $\gamma\left[a_{i_{m}}^{(j)}\right]=\left\{a_{i_{m}}^{(j)}\right\}$ is a minimal element for $\leqslant$.

Lemma 3.4. Let $\sigma: x \Rightarrow y$ be a transition in $\Pi_{i \in I}^{*} S_{i}$. A maximal element for the partial ordering $\leqslant$ defined above on $A_{x}^{\sigma}$ consists of consecutive elements $a_{i_{m}}^{(j)}$ of the decomposition (1) of $x$, all contained in a single syllable of $x$.

Proof. Suppose $a_{i_{m}}^{(j)} \gamma a_{i_{m}}^{\left(j_{1}\right)}$ and $a_{i_{n}}^{(k)} \gamma a_{i_{n}}^{\left(k_{1}\right)}$. If these four elements of $A_{x}^{\sigma}$ have the disposition $\cdots a_{i_{m}}^{(j)} \cdots a_{i_{n}}^{(k)}{ }^{n} \cdots a_{i_{m}}^{\left(j_{1}\right)} \cdots a_{i_{n}}^{\left(k_{1}\right)} \ldots$ in the decomposition (1) of $x$, then the common modification of $a_{i_{m}}^{(j)}$ and $a_{i_{m}}^{\left(j_{1}\right)}$ occurs at the same step of $\sigma$ as the common modification of $a_{i_{n}}^{(k)}$ and $a_{i_{n}}^{\left(k_{1}\right)}$. It follows that all four elements are in the same class of $\gamma$. If the four elements have the disposition $\cdots a_{i_{m}}^{(j)} \cdots a_{i_{n}}^{(k)} \cdots a_{i_{n}}^{\left(k_{1}\right)} \cdots a_{i_{m}}^{\left(j_{1}\right)} \cdots$, then the common modification of $a_{i_{n}}^{(k)}$ and $a_{i_{n}}^{\left(k_{1}\right)}$ takes place at a step of $\sigma$ occurring before the step modifying $a_{i_{m}}^{(j)}$ and $a_{i_{m}}^{\left(j_{1}\right)}$. But when $a_{i_{m}}^{(j)}$ and $a_{i_{m}}^{\left(j_{1}\right)}$ are modified they belong to a syllable of the form $x_{i_{m}} a_{i_{m}}^{(j)} y_{i_{m}} a_{i_{m}}^{\left(j_{1}\right)} z_{i_{m}}$ (one or both of $x_{i_{m}}, z_{i_{m}}$ are eventually empty symbols), and this syllable is a descendant of $a_{i_{n}}^{(k)}$. By the definition of $\leqslant$, we have $\gamma\left[a_{i_{m}}^{(j)}\right]$ $\leqslant \gamma\left[a_{i_{n}}^{(k)}\right]$. If $\gamma\left[a_{i_{m}}^{(j)}\right]$ is a maximal element of $A_{x}^{\sigma}$, we deduce $\gamma\left[a_{i_{m}}^{(j)}\right]=\left[a_{i_{n}}^{(k)}\right]$ and the lemma follows.

Lemma 3.5. Let $\sigma: x \Rightarrow y$ be a transition in $\Pi_{i \in I}^{*} S_{i}$, with $x \in S_{i}, y \in S_{j}$ (eventually $i=j$ ). Then there is a connected transition $\tau: x \Rightarrow y$ in $\prod_{i \in I}^{*} S_{i}$.

Proof. By induction on the length (i.e. the number of steps) of $\sigma$. The result is clear if $\sigma$ has length 1 . We assume it is true for any transition of length 
strictly less than the length of $\sigma$. The factorization (1) of $x$ is $x=u_{i}$ or

$$
x=u_{i}^{(1)} a_{i}^{(1)} u_{i}^{(2)} a_{i}^{(2)} \cdots u_{i}^{(n)} a_{i}^{(n)} u_{i}^{(n+1)} .
$$

The case $x=u_{i}$ occurs when the first step of $\sigma$ is an $S$-step, i.e. $\sigma$ has the form $x=u_{i} \rightarrow u_{k} \Rightarrow y$. The induction hypothesis applied to the transition $u_{k} \Rightarrow y$ gives the result. Suppose $x$ has the factorization $\left(1^{\prime}\right)$ with $n \geqslant 1$. If all the elements $a_{i}^{(1)}, \cdots, a_{i}^{(n)}$ are unmodified by $\sigma$ (i.e. are minimal singleton $\gamma$-classes), then $y \in S_{i}$ and there are transitions in $\sigma$ such that $u_{i}^{(k)} \Rightarrow b_{i}^{(k)}$ for every $k, 1 \leqslant$ $k \leqslant n+1$. By the induction hypothesis there are connected transitions $\tau^{(k)}: u_{i}^{(k)}$ $\Rightarrow b_{i}^{(k)}$, and $\tau^{(1)}, \tau^{(2)}, \cdots, \tau^{(k+1)}$ applied successively give a connected transition $\tau: x \Rightarrow y$. If there are elements $a_{i}^{(1)}, \cdots, a_{i}^{(n)}$ modified by $\sigma$, then by Lemma 3.4 there is a segment of $x$, say

$$
x^{\prime}=u_{i}^{(k)} a_{i}^{(k)} u_{i}^{(k+1)} a_{i}^{(k+1)} \cdots u_{i}^{(k+l)} a_{i}^{(k+l)} a_{i}^{(k+l)} a_{i}^{(k+l)} u_{i}^{(k+l+1)},
$$

such that the elements $a_{i}^{(k)}, a_{i}^{(k+1)}, \cdots, a_{i}^{(k+1)}$ form a maximal $\gamma$-class of $A_{x}^{\sigma}$. Since $\gamma\left(a_{i}^{(k)}\right)$ is maximal, all the steps of $\sigma$ affecting $x^{\prime}$ until the modifying step common to $a_{i}^{(k)}, \cdots, a_{i}^{(k+l)}$ can be performed at the beginning of $\sigma$ in the order in which they appear in $\sigma$. Thus $\sigma$ has the same length as the transition

$$
\begin{aligned}
& x=u_{i}^{(1)} a_{i}^{(1)} \cdots a_{i}^{(k-1)} x^{\prime} a_{i}^{(k+1+1)} \cdots a_{i}^{(n)} u_{i}^{(n+1)} \\
& \quad \stackrel{\sigma_{1}}{\Rightarrow} u_{i}^{(1)} a_{i}^{(1)} \cdots a_{i}^{(k-1)} x^{\prime \prime} a_{i}^{(k+l+1)} \cdots a_{i}^{(n)} u_{i}^{(n+1)} \\
& \quad \stackrel{\sigma_{2}}{\Rightarrow} y
\end{aligned}
$$

where the step following immediately the syllable containing $x^{\prime \prime}$ is the modifying step common to $a_{i}^{(k)}, \cdots, a_{i}^{(k+l)}$. The induction hypothesis applied to $\sigma_{1}$ and $\sigma_{2}$ gives a connected transition $\tau: x \Rightarrow y$. This completes the proof of Lemma 3.5 , and thus Theorem 3.3 is established.

As a consequence of Theorem 3.3 we deduce Howie's result [5].

CoRollary 3.6. Let $\left[S_{i} ; U ; h_{i}\right]_{i \in I}$ be a semigroup amalgam. If $U_{i}=h_{i}(U)$ is almost unitary in $S_{i}$ for every $i \in I$, then the amalgam is embeddable.

Proof. Recall that the hypothesis $U_{i}$ almost unitary in $S_{i}$ for every $i \in I$ (see [2, p. 144]), means in particular that for every $i \in I$ there is a pair of linked left and right translations $\lambda_{i}, \rho_{i}$ of $S_{i}$ such that $U_{i}$ is unitary in $\lambda_{i} S_{i} \rho_{i}$. Denoting $\lambda_{i}\left(s_{i}\right) \rho_{i}$ by $\theta^{i}\left(s_{i}\right)$, we also have $\theta^{i}\left(s_{i} u_{i} t_{i}\right)=\theta^{i}\left(s_{i}\right) u_{i} \theta^{i}\left(t_{i}\right)$, for every $s_{i}, t_{i} \in S_{i}, u_{i} \in$ $U_{i}, s_{i}$ or $t_{i}$ being eventually empty symbols (see [2, Lemma 9.36]). In what follows, some of the elements $\theta^{i}\left(s_{i}\right)$ are elements of $U_{i}$. We denote them by $\theta_{i}^{i}\left(s_{i}\right)$ and define $\theta_{j}^{i}\left(s_{i}\right)$ by $\theta_{j}^{i}\left(s_{i}\right)=h_{j}\left[h_{i}^{-1} \theta^{i}\left(s_{i}\right)\right]$. Given a balanced system $\Sigma$ over $\left[S_{i} ; U ; h_{i}\right]_{i \in I}$ we prove that the locked equation or the locked sentence holds, as follows. Let $i_{0}$ be the index of the locked equation in case $\Sigma$ is of type $\mathrm{I}$; in this 
case we will show that all $s$-variables $s_{i}, i \neq i_{0}$, appearing in $\Sigma$ are such that $\theta^{i}\left(s_{i}\right)$ $\in U_{i}$. Similarly let $i_{0}, j_{0}$ be the indices appearing in the locked sentence in case $\Sigma$ is of the type II; in this case we show that all s-variables $s_{i}, i \neq i_{0}$ and $i \neq j_{0}$, appearing in $\Sigma$ are such that $\theta^{i}\left(s_{i}\right) \in U_{i}$, and that all s-variables in one of any two consecutive equations with indices $i_{0}$ and $j_{0}$ are such that $\left.\theta^{i}{ }^{i} s_{i_{0}}\right) \in U_{i_{0}}$ (or $\left.\theta^{j_{0}}\left(s_{j_{0}}\right) \in U_{j_{0}}\right)$. If $\Sigma$ is of type I, the locked equation is derived from $\Sigma$ by replacing each step of the connected sequence of $\Sigma$ by an equality in $S_{i_{0}}$. This is possible since each equation of $\Sigma$ with index $i \neq i_{0}$ can be replaced by an equation with variables in $U_{i}$ and thus in $U_{i_{0}}$. For example the equation $s_{i}^{(k)} u_{i}^{(l)} s_{i}^{(m)}$ $=s_{i}^{(n)} u_{i}^{(l+1)}$ can be replaced by $\theta_{i_{0}}^{i}\left(s_{i}^{(k)}\right) u_{i_{0}}^{(l)} \theta_{i_{0}}^{i}\left(s_{i}^{(m)}\right)=\theta_{i_{0}}^{i}\left(s_{i}^{(n)}\right) u_{i_{0}}^{(l+1)}$. A similar argument applies if $\Sigma$ is a system of type II, but in this case we need the additional property that all the $s$-variables in the initial or in the final equation of $\Sigma$ (with respective indices $i_{0}$ and $j_{0}$ ) are such that their image under $\theta^{i_{0}}$ or $\theta^{j_{0}}$ are in $U_{i_{0}}$ or $U_{j_{0}}$. In order to prove all the required properties of elements of the form $\theta^{i}\left(s_{i}\right)$ where $s_{i}$ is an $s$-variable in $\Sigma$, we transform $\Sigma$ into a system of equations over the amalgam $\left[\theta^{i}\left(S_{i}\right) ; U ; h_{i}\right]_{i \in I}$ by applying $\theta^{i}$ to both sides of any equation in $\theta$ with index $i$. It is thus enough to show that the properties indicated for elements $\theta^{i}\left(s_{i}\right)$ are in fact true for $s$-variables in a balanced system $\Sigma$ over an amalgam such that $U_{i}$ is unitary in $S_{i}$ for every $i \in I$. This is done by induction on the number of equations in $\Sigma$, the idea of the induction being the possibility of moving forward the first $S$-step in the connected transition defined by $\Sigma$ (cf. proof of Proposition 2.1). If $\Sigma$ is not empty, then the connected transition of $\Sigma$ contains at least one $E$-step or one $E_{r}$-step followed by an $S$-step. Thus $\Sigma$ contains two consecutive equations of the form

$$
\begin{aligned}
\cdots & =u_{i}^{(k)} s_{i}^{(l)} \\
s_{j}^{(m)} u_{j}^{(k)} & =u_{j}^{(k+1)}
\end{aligned}
$$

or

$$
\begin{aligned}
\cdots & =s_{i}^{(l)} u_{i}^{(k)} \\
u_{j}^{(k)} s_{j}^{(m)} & =u_{j}^{(k+1) .} .
\end{aligned}
$$

In both cases it follows that $s_{j}^{(m)}$ is an element of $U_{j}$ which is denoted by $\tau_{j}\left(s_{j}^{(m)}\right)$. Also, we put $\tau_{i}\left(s_{j}^{(m)}\right)=h_{i}\left[h_{j}^{-1} \tau_{j}\left(s_{j}^{(m)}\right)\right]$. We construct a balanced system $\Sigma^{\prime}$ containing less equations than $\Sigma$ by deleting the equation $s_{j}^{(m)} u_{j}^{(k)}=u_{j}^{(k+1)}$ ) (or $\left.u_{j}^{(k)} s_{j}^{(m)}=u_{j}^{(k+1)}\right)$ in the first succession of equations of the form (1) or (2) in $\Sigma$ and by modifying correspondingly the remaining equations. To indicate the details of these modifications, suppose we suppress equation (1.b) (the case (2.b) is symmetrical). If the index of the equation following immediately (1.b) in $\Sigma$ is 
distinct from $i$ (index of (1.a)) the equations of $\Sigma$ following (1.b) are not modified. Equation (1.a) is replaced by

$$
\tau_{i}\left(s_{j}^{(m)}\right) \cdots=u_{i}^{(k+1)} s_{i}^{(l)}
$$

which is derived from (1.a) by multiplication of both sides on the left by $\tau_{i}\left(s_{j}^{(m)}\right)$. All equations preceding (1.a) are similarly multiplied on the left by $\tau_{k}\left(s_{j}^{(m)}\right)$ which can alternatively be considered as an $s$-variable or a $u$-variable, until an equation containing eventually the variable $s_{i}^{(l)}$ is reached. We replace $s_{i}^{(m)}$ by $\tau_{i}\left(s_{i}^{(m)}\right)$ in it and continue the multiplication process if necessary in other equations so as to obtain a balanced system $\Sigma^{\prime}$. In case the index of the equation following (1.b) in $\Sigma$ is the index of (1.a), then this equation has the form $\cdots u_{i}^{(k+1)} s_{i}^{(l)}=\cdots$. We replace (1.a) and the equation following (1.b) by a single equation obtained by replacing $u_{i}^{(k+1)} s_{i}^{(l)}$ in the latter by its value $\tau_{i}\left(s_{j}^{(m)}\right) \cdots$ given in (1.a'). Other modifications are performed as indicated above, yielding again a system $\Sigma^{\prime}$ with less equations than $\Sigma$. By the induction hypothesis, all $s$-variables in $\Sigma^{\prime}$ have the required properties. But $s$-variables in $\Sigma^{\prime}$ are either $s$-variables in $\Sigma$ or products of $s$-variables in $\Sigma$ by an element in one of the $U_{i}$ 's. The unitary condition implies then that all $s$-variables of $\Sigma$ in $\Sigma^{\prime}$ have the required properties, while variables of $\Sigma$, not in $\Sigma^{\prime}$, are in one of the $U_{i}$ 's by the reduction process. This completes the proof of Corollary 3.6.

In contrast to the previous result, Theorem 1.4 is an immediate consequence of Theorem 3.3. Indeed, the hypothesis in an ideal amalgamation is such that the sentence locked to a balanced system is directly derivable from the system itself.

It does not seem possible to derive Proposition 2.4 , or the embeddability property in the inverse semigroup case, without further analysis of balanced systems in these particular cases. This leads to proofs that are, at the present state of affairs, longer than the original ones.

4. Finite sets of equational implications. In order to define equational implications over a semigroup amalgam we need the following

DEFInItion 4.1. A morphism from the semigroup amalgam $\left[S_{i} ; U ; h_{i}\right]_{i \in I}$ to the amalgam $\left[T_{i} ; V ; k_{i}\right]_{i \in I}$ is a collection of homomorphisms $\left[\Phi_{i} ; \Phi\right]_{i \in I}$ such that the diagrams

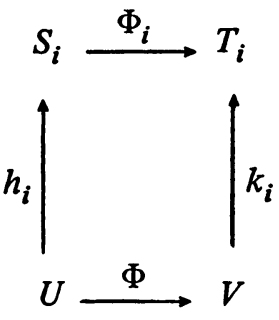

commute for every $i \in I$. 
It is easily seen that the existence of a morphism $\left[\Phi_{i}, \Phi\right]_{i \in I}$ is equivalent to the existence of a homomorphism $\theta: \Pi_{i \in I^{U}}^{*} S_{i} \rightarrow \Pi_{i \in I^{V}}^{*} T_{i}$ such that $\theta\left[\varphi_{i}\left(S_{i}\right)\right]$ $\subseteq \bar{\varphi}_{i}\left(T_{i}\right)$ and $\theta[h(U)] \subseteq k[V]$ with $\varphi_{i}$ [resp. $\left.\bar{\varphi}_{i}\right]$ the canonical homomorphism $S_{i}$ $\rightarrow \Pi_{U}^{*} S_{i}$ [resp. $T_{i} \rightarrow \Pi_{V}^{*} T_{i}$ ] and $h$ [resp. $k$ ] the canonical homomorphism $U \rightarrow$ $\Pi_{U}^{*} S_{i}$ [resp. $V \rightarrow \Pi_{V}^{*} T_{i}$ ] (see $\left.\S 1\right)$.

Let $\Sigma_{i}(i \in I)$ and $\Omega$ be alphabets (i.e. sets of letters) such that there are injections $\chi_{i}: \Omega \rightarrow \Sigma_{i}$ for every $i \in I$. Extend $\chi_{i}$ to an injective homomorphism, also denoted $\chi_{i}$ from $\Omega^{*}$ to $\Sigma_{i}^{*}$ where $\Omega^{*}$ and $\Sigma_{i}^{*}$ are the free semigroups on $\Omega$ and $\Sigma_{i}$ respectively. Then $\left[\Sigma_{i}^{*} ; \Omega^{*} ; \chi_{i}\right]_{i \in I}$ is an embeddable semigroup amalgam.

Let $w_{i}^{\left(k_{p}\right)}, w_{i}^{\left(l_{p}\right)}, v_{i}^{\left(m_{q}\right)}, v_{i}^{\left(n_{q}\right)} \in \Sigma_{i}^{*}$ with $p, q=1,2, \cdots$.

DEFINITION 4.2. We say that the set of equational implications

(*) $w_{i}^{\left(k_{p}\right)}=w_{i}^{\left(l_{p}\right)}(i \in I, p=1,2, \cdots)$ imply $v_{i}^{\left(m_{q}\right)}=v_{i}^{\left(n_{q}\right)}(i \in I, q=1,2, \cdots)$

holds in the semigroup amalgam $\left[S_{i} ; U ; h_{i}\right]_{i \in I}$ if for any morphism $\left[\Phi_{i} ; \Phi\right]_{i \in I}$ : $\left[\Sigma_{i}^{*} ; \Omega^{*} ; \chi_{i}\right]_{i \in I} \rightarrow\left[S_{i} ; U ; h_{i}\right]_{i \in I}$

$$
\begin{aligned}
& \Phi_{i}\left(w_{i}^{\left({ }^{k}\right.}{ }^{p}\right)=\Phi_{i}\left(w_{i}^{\left(l^{p}\right)}\right)(i \in I, p=1,2, \cdots) \text { imply } \\
& \Phi_{i}\left(v_{i}^{\left(m_{q}\right)}\right)=\Phi_{i}\left(v_{i}^{\left(n_{q}\right)}\right)(i \in I, q=1,2, \cdots) .
\end{aligned}
$$

It should be noted that the equational implications (*) contain in general existential quantifiers affecting elements of $\Omega^{*}$ involved in the expressions of $w_{i}^{\left(k_{p}\right)}, w_{i}^{\left(l_{p}\right)}, v_{i}^{\left(m_{q}\right)}, v_{i}^{\left(n_{q}\right)}$. As an example to write

$$
\begin{aligned}
& u_{1}^{(1)} s_{1}^{(1)}=s_{1}^{(2)} u_{1}^{(2)} \\
& u_{2}^{(2)}=s_{2}^{(1)} u_{2}^{(3)} \\
& s_{1}^{(1)} u_{1}^{(2)}=u_{1}^{(4)} \\
& \text { implies } \\
& s_{2}^{(1)}=u_{2}^{(4)}
\end{aligned}
$$

means: If there exists $u^{(1)}, u^{(2)}, u^{(3)} \in \Omega^{*}$ such that $\chi_{1}\left(u^{(1)}\right) s_{1}^{(1)}=s_{1}^{(2)} \chi_{1}\left(u^{(2)}\right)$, $\chi_{2}\left(u^{(2)}\right)=s_{2}^{(1)} \chi_{2}\left(u^{(3)}\right)$, then there exists $u^{(4)} \in \Omega^{*}$ such that $s_{1}^{(1)} \chi_{1}\left(u^{(2)}\right)=$ $\chi_{1}\left(u^{(4)}\right), s_{2}^{(1)}=\chi_{2}\left(u^{(4)}\right)$.

We shall show that no finite set of equational implications can serve as a necessary and sufficient condition for a semigroup amalgam to be embeddable. As for the embedding of a semigroup into a group [8], the idea is to show that given any finite set of equational implications, holding in any embeddable semigroup amalgam, it is possible to construct a nonembeddable amalgam in which the implications hold. We construct a nonembeddable amalgam $\mathfrak{X}_{n}=\left[S_{i} ; U ; h_{i}\right]_{i \in I}$ with card $I=3$ the construction being easily extendible to any finite set $I$. The semigroup $U$ is the free semigroup on $u^{(1)}, u^{(2)}, \cdots, u^{(4 n+4)}$. The semigroup $S_{i}$ $(i=1,2,3)$ is generated by elements $u_{i}^{(1)}, u_{i}^{(2)}, \cdots, u_{i}^{(4 n+4)}$ together with all 
the elements $s_{i}^{(k)}$ appearing in the set $\Pi\left(\mathscr{H}_{n}\right)$ of generating relations indicated in the following board.

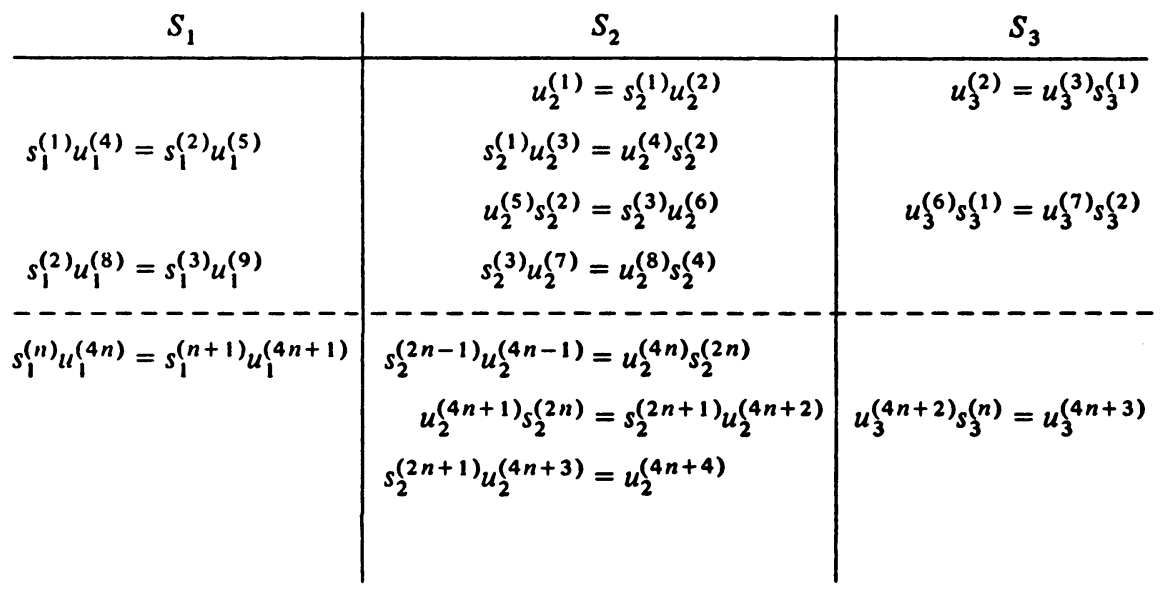

The general form of a relation for $S_{1}$ is $s_{1}^{(k)} u_{1}^{(4 k)}=s_{1}^{(k+1)} u_{1}^{(4 k+1)}(1 \leqslant k \leqslant n)$.

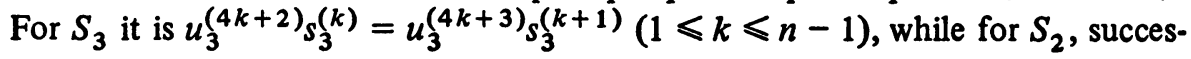
sive relations are

$$
\begin{aligned}
s_{2}^{(k)} u_{2}^{(2 k+1)} & =u_{2}^{(2 k+2)} s_{2}^{(k+1)}, \\
u_{2}^{(2 k+3)} s_{2}^{(k+1)} & =s_{2}^{(k+2)} u_{2}^{(2 k+4)}, \quad 1 \leqslant k \leqslant 2 n-1 .
\end{aligned}
$$

In each of $S_{1}, S_{2}, S_{3}$ the word problem is easily solved: Every word can be put into a unique canonical form by lowering, if possible, the superscript of $u_{i}^{(n)}$. For example, in $S_{2}$ replace occurrences of $s_{2}^{(1)} u_{2}^{(2)}$ by $u_{2}^{(1)}, u_{2}^{(2 k+2)} s_{2}^{(2 k+2)}$ by $s_{2}^{(k)} u_{2}^{(2 k+1)}$, etc. Due to the independence of the presentation relations, these replacements can be performed at most once for every occurrence of $u_{2}^{(n)}$. It follows that $h_{i}(U)$ is free in $S_{i}$ and thus that $\mathscr{U}_{n}$ is a semigroup amalgam. Furthermore $\mathfrak{Q}_{n}$ is not embeddable, since the set of all presentation relations constitutes a balanced system over $\mathfrak{U}_{n}$, with locked equation $s_{1}^{(1)} u_{1}^{(1)}=s_{1}^{(n)} u_{1}^{(4 n+4)}$. The locked equation does not hold in $S_{1}$, for $s_{1}^{(1)} u_{1}^{(1)}$ and $s_{1}^{(n)} u_{1}^{(4 n+4)}$ are both in canonical forms.

LEMmA 4.3. Let $\mathfrak{U}_{n}=\left[S_{i} ; U ; h_{i}\right]_{i \in I}$ be the semigroup amalgam where the semigroups $S_{i}$ have the presentation relations $\Pi\left(\mathscr{U}_{n}\right)$ indicated above, and let $\Pi$ be a proper subset of $\Pi\left(\mathfrak{U}_{n}\right)$. For every $i \in I$, denote by $S_{i}^{\Pi}$ the semigroup having the same generators as $S_{i}$ and presentation relations all the relations with index $i$ contained in $\Pi$. Then the amalgam $\mathscr{U}_{n}^{\Pi}=\left[S_{i}^{\Pi} ; U ; h_{i}\right]_{i \in I}$ is embeddable.

Proof. We show that condition (iv) of Theorem 1.1 holds in $\mathfrak{Q}_{n}^{\Pi}$. By 
Lemma 3.5 it is enough to consider connected transitions $\tau: x \Rightarrow y$ with $x \in S_{i}^{\Pi}$, $y \in S_{i}^{\Pi}$. If $x$ does not contain any occurrence of $u_{i}^{(1)}, u_{i}^{(2)}, u_{i}^{(4 n+3)}$ or $u_{i}^{(4 n+4)}$, then $\tau$ is either empty (in case $i=j$ ) and $x=y$ or $\tau$ consists of a single $S$-step (in case $i \neq j$ ) performed on $x \in h_{i}(U)$. A similar result holds in case $x$ contains occurrences of $u_{i}^{(k)}$ with $k=1,2,4 n+3,4 n+4$. In this case, possible successive steps of $\tau$ are indicated by the factorizations given by the relations in $\Pi$. For example, if $x=x_{i}^{(1)} u_{i}^{(1)} x_{i}^{(2)}$ the possibility for $\tau$ is

$$
\begin{aligned}
x_{i}^{(1)} u_{i}^{(1)} x_{i}^{(2)} & \rightarrow x_{i}^{(1)} u_{2}^{(1)} x_{i}^{(2)}=x_{i}^{(1)} s_{2}^{(1)} u_{2}^{(2)} x_{i}^{(2)} \\
& \rightarrow x_{i}^{(1)} s_{2}^{(1)} u_{3}^{(2)} x_{i}^{(2)}=x_{i}^{(1)} s_{2}^{(1)} u_{3}^{(3)} s_{3}^{(1)} x_{i}^{(2)} \rightarrow \text { etc. }
\end{aligned}
$$

But such a sequence does not yield an element $y \in S_{j}^{\Pi}$ in case $i \neq 1$, and also in case $i=1$ due to the fact that $\Pi$ is a proper subset of $\Pi\left(\mathscr{U}_{n}\right)$.

THEOREM 4.4. Given any finite set of equational implications, each of which holds in every embeddable semigroup amalgam, there is an integer $n$ and a nonembeddable semigroup amalgam $\mathfrak{Q}_{n}$ such that the given implications hold in $\mathfrak{U}_{n}$.

Proof. Let the finite set of equational implications be:

$$
\begin{aligned}
& w_{i}^{\left(k_{p}\right)}=w_{i}{ }^{\left(l_{p}\right)}(i \in I, p=1,2, \cdots) \text { imply } \\
& v_{i}^{\left(m_{q}\right)}=v_{i}^{\left(n_{q}\right)}(i \in I, q=1,2, \cdots) .
\end{aligned}
$$

Let $n$ be an integer greater than the total length of all words $w_{i}^{\left(k_{p}\right)}, w_{i}^{\left(l_{p}\right)}$ appearing in the equational implications above. Then all the words in these implications can be considered as words in the amalgam $F_{n}=\left[\Sigma_{i}^{*}, \Omega^{*}, \chi_{i}\right]_{i \in I}$ where $\Omega=$ $\left\{u^{(1)}, u^{(2)}, \cdots, u^{(4 n+4)}\right\}$ and $\Sigma_{i}=\left\{s_{i}^{(1)}, s_{i}^{(2)}, \cdots, u_{i}^{(1)}, u_{i}^{(2)}, \cdots, u_{i}^{(4 n+4)}\right\}$. Let $\left[\Phi_{i}, \Phi\right]_{i \in I}$ be a morphism from $F_{n}$ to $\mathfrak{R}_{n}$, such that $\Phi_{i}\left(w_{i}^{\left(k_{p}\right)}\right)=\Phi_{i}\left(w_{i}^{\left(l_{p}\right)}\right)$ for $i \in I, p=1,2, \cdots$. Suppose $w_{i}^{\left(k_{p}\right)}=x_{i}^{\left(k_{2}\right)} \cdots x_{i}^{\left(k_{r}\right)}$ and $w_{i}^{\left(l_{p}\right)}=x_{i}^{\left(l_{1}\right)} x_{i}^{\left(l_{2}\right)}$ $\cdots x_{i}^{\left(l_{s}\right)}$ are the expressions of $w_{i}^{\left(k_{p}\right)}$ and $w_{i}^{\left(l_{p}\right)}$ as products of elements of $\Sigma$. Then

$$
\text { (1) } \Phi_{i}\left[x_{i}^{\left(k_{1}\right)}\right] \Phi_{i}\left[x_{i}^{\left(k_{2}\right)}\right] \cdots \Phi_{i}\left[x_{i}^{\left(k_{r}\right)}\right]=\Phi_{i}\left[x_{i}^{\left(l_{1}\right)}\right] \Phi_{i}\left[x_{i}^{\left(l_{2}\right)}\right] \cdots \Phi_{i}\left[x_{i}^{\left(l_{s}\right)}\right] \text {. }
$$

If we assume that each $\Phi_{i}\left[x_{i}^{(k)}\right]$ and $\Phi_{i}\left[x_{i}^{(l)}\right]$ is in its canonical form in $\vartheta_{n}$, then by the choice of $n$,to transform the left sides of all the equalities (1) for $i \in I$, $p=1,2, \cdots$ into the corresponding right sides we will need a proper subset $\Pi$ of the relations $\Pi\left(\vartheta_{n}\right)$. It follows that the morphism $\left[\Phi_{i}, \Phi\right]_{i \in I}$ induces a morphism $\left[\psi_{i} ; \psi\right]_{i \in I}: F_{n} \rightarrow \vartheta_{n}^{\Pi}$ such that the diagram below, with $\left[\theta_{i}, \theta\right]_{i \in I}$ the canonical morphism: $\mathfrak{Q U}_{n}^{\Pi} \rightarrow \mathfrak{U}_{n}$ 


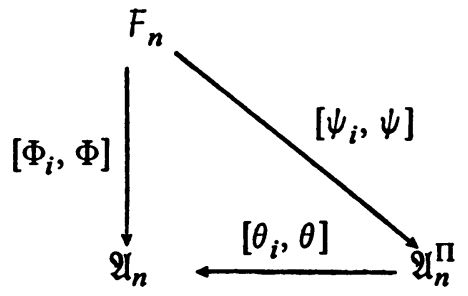

commutes. From (1) we deduce $\psi_{i}\left(w_{i}^{\left(k_{p}\right)}\right)=\psi_{i}\left(w_{i}^{\left(l_{p}\right)}\right)$ for every $i \in I, p=1,2$, -.. By Lemma 3.3 and the hypothesis on the equational implications we deduce $\psi_{i}\left(v_{i}^{\left(m_{q}\right)}=\psi_{i}\left(v_{i}^{\left(n_{q}\right)}\right)\right.$ and thus $\Phi_{i}\left(v_{i}^{\left(m_{q}\right)}\right)=\Phi_{i}\left(v_{i}^{\left(n_{q}\right)}\right)$ for every $i \in I, p=1,2$, $\cdots$, showing that the given implications hold in $\mathfrak{U}_{n}$.

\section{REFERENCES}

1. N. Bourbaki, Eléments de mathématique, Algèbre, Chaps. 1-3, Hermann, Paris, 1970. MR 43 \#2.

2. A. H. Clifford and G. B. Preston, The algebraic theory of semigroups. Vol. II, Math. Surveys, no. 7, Amer. Math. Soc., Providence, R. I., 1967. MR 36 \#1558.

3. P.-A. Grillet and M. Petrich, Free products of semigroups amalgamating an ideal, J. London Math. Soc. (2) 2 (1970), 389-392. MR 43 \#2132.

4. T. E. Hall, Inverse semigroups and the amalgamation property (to appear).

5. J. M. Howie, Embedding theorems with amalgamation for semigroups, Proc. London Math. Soc. (3) 12 (1962), 511-534. MR 25 \#2139.

6. E. S. Ljapin, Independent intersection of subsemigroups of a semigroup, Izv. Vyš̌. Učebn. Zaved. Matematika 4 (1970), 67-73. (Russian)

7. A. I. Malcev, On the immersion of associative systems in groups, Mat. Sb. 6 (1939), 331-336. (Russian) MR 2, 7.

8. - On the immersion of associative systems in groups. II, Mat. Sb. 8 (1940), 251-264. (Russian) MR 2, 128.

DEPARTMENT OF MATHEMATICS, PENNSYLVANIA STATE UNIVERSITY, UNIVERSITY PARK, PENNSYLVANIA 16802 